OPEN ACCESS

Edited by:

Jesus Avila,

Universidad Autonoma de Madrid,

Spain

Reviewed by:

Jaime Gomez-Ramirez,

Fundacion Centro De Investigacion De Enfermedades Neurologicas, Spain

Pierpaolo Sorrentino,

Università degli Studi di Napoli

Parthenope, Italy

*Correspondence:

Inmaculada C. Rodríguez-Rojo inmaculada.rodriguez@ctb.upm.es

tThese authors have contributed equally to this work and share first authorship

Specialty section: This article was submitted to Neurodegeneration, a section of the journal

Frontiers in Neuroscience

Received: 28 May 2018 Accepted: 11 September 2018 Published: 02 October 2018

Citation:

Rodríguez-Rojo IC, Cuesta P,

López ME, de Frutos-Lucas J, Bruña R, Pereda E, Barabash A, Montejo P, Montenegro-Peña $M$,

Marcos A, López-Higes $R$, Fernández A and Maestú F (2018) BDNF Val66Met Polymorphism and Gamma Band Disruption in Resting State Brain Functional Connectivity: A Magnetoencephalography Study in Cognitively Intact Older Females. Front. Neurosci. 12:684. doi: 10.3389/fnins.2018.00684

\section{BDNF Val66Met Polymorphism and Gamma Band Disruption in Resting State Brain Functional Connectivity: A Magnetoencephalography Study in Cognitively Intact Older Females}

\author{
Inmaculada C. Rodríguez-Rojo ${ }^{1,2 * t}$, Pablo Cuesta ${ }^{1,3 \dagger}$, María Eugenia López ${ }^{1,2}$, \\ Jaisalmer de Frutos-Lucas ${ }^{1,4}$, Ricardo Bruña ${ }^{1,2,5}$, Ernesto Pereda ${ }^{1,3}$, Ana Barabash ${ }^{6,7}$, \\ Pedro Montejo ${ }^{8}$, Mercedes Montenegro-Peña ${ }^{8}$, Alberto Marcos ${ }^{9}$, Ramón López-Higes ${ }^{2}$, \\ Alberto Fernández ${ }^{1,10}$ and Fernando Maestú ${ }^{1,2,5}$ \\ ${ }^{1}$ Laboratory of Cognitive and Computational Neuroscience, Center for Biomedical Technology, Universidad Complutense \\ and Universidad Politécnica de Madrid, Madrid, Spain, ${ }^{2}$ Department of Experimental Psychology, Cognitive Processes and \\ Speech Therapy, Universidad Complutense de Madrid, Madrid, Spain, ${ }^{3}$ Electrical Engineering and Bioengineering Lab, \\ Department of Industrial Engineering and IUNE, Universidad de La Laguna, Tenerife, Spain, ${ }^{4}$ Biological and Health \\ Psychology Department, Universidad Autónoma de Madrid, Madrid, Spain, ${ }^{5}$ Networking Research Center on \\ Bioengineering, Biomaterials and Nanomedicine (CIBER-BBN), Madrid, Spain, ${ }^{6}$ Laboratory of Psychoneuroendocrinology \\ and Genetics, Hospital Clínico San Carlos, Madrid, Spain, ${ }^{7}$ Instituto de Investigación Sanitaria del Hospital Clínico San Carlos \\ (IdISSC), Madrid, Spain, ${ }^{8}$ Center for the Prevention of Cognitive Impairment, Public Health Institute, Madrid-Salud, Madrid, \\ Spain, ${ }^{9}$ Neurology Department, Hospital Clínico San Carlos, Madrid, Spain, ${ }^{10}$ Department of Legal Medicine, Psychiatry, and \\ Pathology, Universidad Complutense de Madrid, Madrid, Spain
}

The pathophysiological processes undermining brain functioning decades before the onset of the clinical symptoms associated with dementia are still not well understood. Several heritability studies have reported that the Brain Derived Neurotrophic Factor $(B D N F)$ Val66Met genetic polymorphism could contribute to the acceleration of cognitive decline in aging. This mutation may affect brain functional connectivity (FC), especially in those who are carriers of the BDNF Met allele. The aim of this work was to explore the influence of the BDNF Val66Met polymorphism in whole brain eyes-closed, resting-state magnetoencephalography (MEG) FC in a sample of 36 cognitively intact (Cl) older females. All of them were $\varepsilon 3 \varepsilon 3$ homozygotes for the apolipoprotein $E(A P O E)$ gene and were divided into two subgroups according to the presence of the Met allele: Val/Met group $(n=16)$ and Val/Val group $(n=20)$. They did not differ in age, years of education, Mini-Mental State Examination scores, or normalized hippocampal volumes. Our results showed reduced antero-posterior gamma band $\mathrm{FC}$ within the Val/Met genetic risk group, which may be caused by a GABAergic network impairment. Despite the lack of cognitive decline, these results might suggest a selective brain network vulnerability due to the carriage of the BDNF Met allele, which is linked to a potential progression to dementia. This neurophysiological signature, as tracked with MEG FC, indicates that age-related brain functioning changes could be mediated by the influence of particular genetic risk factors.

Keywords: BDNF Val66Met, magnetoencephalography, gamma rhythms, cognitive functioning, healthy aging, Alzheimer's disease 


\section{INTRODUCTION}

Over the last two decades, the interest in exploring candidate genes related to cognitive and brain aging has significantly increased (Kennedy et al., 2014). The Brain Derived Neurotrophic Factor (BDNF) is one of the genes involved in the aging process due to its protective role in neural function (Chen et al., 2013; Rothman and Mattson, 2013; Marosi and Mattson, 2014; Criscuolo et al., 2015). The molecule derived from the BDNF expression is one of the most abundant brain neurotrophins (Zhang et al., 2007), and its presence is essential for the maintenance, survival, and growth of neurons (CohenCory and Fraser, 1995; Mattson et al., 2004). BDNF is also related to the activity-dependent synaptic plasticity, which is crucial for the modulation of several cognitive domains such as learning, memory, and executive functions (Horch et al., 1999; Egan et al., 2003; Binder and Scharfman, 2004; Bath and Lee, 2006). However, it is well-known that BDNF production decreases with age (Mattson and Magnus, 2006) and more importantly, with the emergence of Alzheimer's Disease (AD)-related neuropathology (Qin et al., 2017). With this background in mind, recent literature (Matyi et al., 2017) has focused on exploring the association between several $B D N F$ single nucleotide polymorphisms (SNPs) and this neurodegenerative disorder, providing additional evidence in support of the interest of further studying this relationship.

Of particular relevance is the role of the Val66Met BDNF SNP, where a Valine (Val) is substituted by a Methionine (Met) at codon 66 (Val66Met, rs6265, G>A) on chromosome 11 (Egan et al., 2003). This functional mutation alters the intracellular trafficking and packaging of proBDNF, affecting the regular activity-dependent expression of the mature BDNF peptide (Egan et al., 2003; Chen et al., 2004). Specifically, Met allele carriers (Val/Met) exhibit a lower BDNF secretion than Val/Val homozygotes (Val/Val) (Kennedy et al., 2014). This alteration has been related to increased hippocampal atrophy (Lim et al., 2013, 2014a; Rabl et al., 2014), loss of white matter integrity (Kennedy and Raz, 2009; Voineskos et al., 2011; Ziegler et al., 2013), greater cognitive decline or higher susceptibility to $A D$ (Voineskos et al., 2011; Kambeitz et al., 2012; Huang et al., 2014; Lim et al., 2014a), and poorer neurocognitive performance in healthy controls (Dincheva et al., 2012).

In addition to genetic approach, the detection of abnormal changes in the brain's oscillatory activity is considered nowadays as one of the most suitable strategies in the study of neurodegenerative disorders (Pievani et al., 2011; Başar and Düzgün, 2016). Functional connectivity (FC) estimators are good examples of such new strategies, since they enable the investigation of the brain from the perspective of a complex neural network. As a consequence, brain diseases are contemplated as network disruption processes (Varela et al., 2001; Stam, 2014). Electroencephalography (EEG) and magnetoencephalography (MEG) are non-invasive neuroimaging techniques that directly measure primary neural activity, and provide useful markers to characterize functional synaptic alterations (Stam, 2010; López et al., 2014; Pievani et al., 2014; Nakamura et al., 2017). BDNF has a regulatory role in the synaptic function, and several studies have reported that the Met allele contributes to the alteration of the morphology and FC in the brain (Beste et al., 2010; Jang et al., 2012; Wei et al., 2012). Therefore, MEG FC emerges as an appropriate tool to assess the effects of $B D N F$ in Met carriers and non-carriers.

Considering the aforementioned, we hypothesize that the carriage of a Met allele (i.e., a lower secretion of BDNF) would produce a disruption in the transmission of brain signals that, in turn, alters FC concomitantly to a poorer cognitive performance. To the best of our knowledge, this is the first study assessing the effect of the BDNF Val66Met SNP in MEG functional brain networks. The multimodal analysis undertaken to test this hypothesis also involved structural (i.e., medial temporal lobe volumes), cognitive (i.e., neuropsychological tests), and $A P O E$ genotyping information from a group of cognitively intact (CI) older females due to their suggested higher predisposition to AD's (Barnes et al., 2005; Fleisher et al., 2005; Neu et al., 2017; Fisher et al., 2018).

\section{MATERIALS AND METHODS}

\section{Participants}

The original sample consisted of $36 \mathrm{CI}$ older Caucasian females recruited from the "Hospital Universitario San Carlos", and the "Center for the Prevention of Cognitive Impairment" of Madrid. All participants underwent an extensive neuropsychological assessment to explore their cognitive functioning, which included the following tests: the Spanish version of the Mini-mental state Examination (MMSE, Lobo et al., 1979), forward and backward digit spam tests (FDS and BDS, Wechsler Memory Scale III, WMS-III; Wechsler, 1997), immediate and delayed recall (IR and DR, WMS-III; Wechsler, 1997), phonemic and semantic fluency ( $\mathrm{PhF}$ and $\mathrm{SF}$, controlled oral word association test; Benton and Hamsher, 1989), Boston naming test (BNT, Kaplan et al., 1983), and trail-making test (TMT) parts A and B (TMT-A and TMT-B, Reitan, 1958). They also underwent a multimodal neuroimaging assessment including Magnetic Resonance Imaging (MRI) and MEG scans. All of them were right-handed, native Spanish speakers and did not differ in age, years of education, MMSE (Folstein et al., 1975; Lobo et al., 1979) score, or normalized hippocampal volumes (see Table 1 for information relative to the characteristics of the participants).

Individuals with any significant medical, neurologic or psychiatric diseases were excluded from the study according to their medical history, neurologic and clinical examination, and MRI results. Inclusion criteria were the absence of significant cerebral-vascular disease (modified Hachinski score $\leq 4$ ) and depressive symptomatology (Yesavage's Depression Scale scores $>9$ ), and an age between 60 and 80 years. In addition, T2weighted MRIs within 12 months before MEG screening should not show any indication of infection, infarction, or focal lesions (rated by two independent experienced radiologists (Bai et al., 2012).

Concerning the genetic load, participant's selection was restricted to those homozygous for the APOE $\varepsilon 3$ allele. Different studies have shown the deleterious effect produced by the 
TABLE 1 | Characteristics of the participants.

\begin{tabular}{lccc}
\hline & $\begin{array}{c}\text { Val/Val } \\
(\boldsymbol{n}=\mathbf{2 0})\end{array}$ & $\begin{array}{c}\text { Val/Met } \\
(\boldsymbol{n}=\mathbf{1 6})\end{array}$ & $\begin{array}{l}\text { Statistics } \\
\text { ( }) \text { value) }\end{array}$ \\
\hline Age & $70.1 \pm 4.8$ & $70.3 \pm 4.9$ & 0.810 \\
Education (years) & $12.6 \pm 4.2$ & $11.3 \pm 5.3$ & 0.453 \\
MMSE & $29.3 \pm 1.0$ & $29.5 \pm 0.7$ & 0.505 \\
BNT & $54.0 \pm 4.3$ & $51.4 \pm 8.4$ & 0.238 \\
Forward digit span & $8.7 \pm 2.6$ & $8.0 \pm 1.5$ & 0.345 \\
Backward digit span & $6.3 \pm 2.1$ & $5.1 \pm 1.7$ & 0.066 \\
Immediate recall & $39.8 \pm 9.9$ & $39.9 \pm 20.1$ & 0.970 \\
Delayed recall & $26.4 \pm 7.3$ & $25.6 \pm 10.0$ & 0.802 \\
Phonemic fluency & $14.6 \pm 3.8$ & $13.9 \pm 4.7$ & 0.656 \\
Semantic fluency & $16.8 \pm 3.8$ & $17.4 \pm 5.0$ & 0.647 \\
TMT-A (time) & $48.4 \pm 13.4$ & $58.3 \pm 24.0$ & 0.125 \\
TMT-B (time) & $107.6 \pm 46.6$ & $138.1 \pm 77.0$ & 0.144 \\
Left hippocampal volume & $0.0027 \pm 0.0003$ & $0.0027 \pm 0.0004$ & 0.987 \\
Right hippocampal & $0.0028 \pm 0.0003$ & $0.0027 \pm 0.0004$ & 0.729 \\
volume & & & \\
Left entorhinal volume & $0.0016 \pm 0.0002$ & $0.0016 \pm 0.0003$ & 0.854 \\
Right entorhinal volume & $0.0017 \pm 0.0003$ & $0.0016 \pm 0.0003$ & 0.232 \\
\hline
\end{tabular}

We present values as mean $\pm S D$. Statistical analyses were performed using one-way ANCOVA (age as confounding covariate).

MMSE, Mini-Mental State Examination; TMT-A, Trail-Making Test part A; TMT-B, TrailMaking Test part B; BNT, Boston Naming Test.

Medial temporal lobe volumes were normalized with respect to the overall intracranial volume to account for differences in head volume over subjects.

combination of being carrier of both the BDNF Met allele and the APOE \&4 allele (Hashimoto et al., 2009; Adamczuk et al., 2013; Kauppi et al., 2014; Lim et al., 2014b). Based on this, we selected only $A P O E \varepsilon 3 \varepsilon 3$ individuals, who in turn were classified as carriers and non-carriers of the BDNF Met allele (i.e., Val/Met vs. Val/Val), since Met/Met homozygotes were not sufficiently prevalent to conduct specific subgroup analysis.

The Hospital Universitario San Carlos local Ethics Committee approved the study, and all the participants provided written informed consent in accordance with the Declaration of Helsinki and prior to their examination to participate in the clinical studies of cognitive impairment.

\section{$B D N F$ and APOE Genotype Test}

DNA was extracted from whole-blood samples of CI older females. As previously described in (Cuesta et al., 2015), APOE haplotype was determined by analyzing SNPs rs7412 and rs429358 genotypes with TaqMan assays using an Applied Biosystems 7900 HT Fast Real Time PCR machine (Applied Biosystems, Foster City, CA). The same procedure was applied to analyze the BDNF rs6265 (Val66Met) SNP. A genotyping call rate over $90 \%$ per plate, sample controls for each genotype and negative sample controls were included in each assay. Three welldifferentiated genotyping clusters for each SNP were required to validate results. Intra and inter-plate duplicates of several DNA samples were included.

\section{Structural MRI}

We collected 3D T1 weighted anatomical brain MRI scans with a General Electric 1.5T MRI scanner, using a highresolution antenna and a homogenization PURE filter (Fast Spoiled Gradient Echo (FSPGR) sequence with parameters: $\mathrm{TR} / \mathrm{TE} / \mathrm{TI}=11.2 / 4.2 / 450 \mathrm{~ms}$; flip angle $12^{\circ} ; 1 \mathrm{~mm}$ slice thickness, a $256 \times 256$ matrix and FOV $25 \mathrm{~cm}$ ). The MRIs were processed with Freesurfer software (version 5.1.0.21) to obtain the volume of gray matter in several brain areas (Fischl et al., 2002), which were normalized with respect to the overall intracranial volume (ICV).

\section{MEG}

\section{Acquisition}

MEG signals were acquired using a whole-head ElektaNeuromag MEG system with 306 channels (Elekta AB, Stockholm, Sweden), placed in a magnetically shielded room (VacuumSchmelze GmbH, Hanua, Germany), at the Center for Biomedical Technology (Madrid, Spain). Participants sat comfortably on a chair with their eyes closed while 4 min of resting state MEG signals data were collected with a sampling frequency of $1,000 \mathrm{~Hz}$ and online band-pass filtered between 0.1 and $330 \mathrm{~Hz}$. The positions of four head-position indicator (HPI) coils attached to the scalp, and each subject's head shape relative to three anatomical locations (nasion and both preauricular points) were defined using a 3D digitizer (Fastrak, Polhemus, VT, USA). The HPI coils continuously controlled the participants' head movements, whereas eye movements were monitored by means of a vertical electrooculograph using a pair of bipolar electrodes. Finally, we supervised the arousal level of each subject through a video camera and checked it via a conversation immediately after the measurement session.

\section{Preprocessing}

In order to remove environmental noise, MEG raw data were first submitted to the Maxfilter software with the temporal extension of the signal space separation method ( $v$ 2.2, correlation threshold $=0.9$, time window $=10 \mathrm{~s}$ ) with movement compensation (Taulu and Simola, 2006). MEG data were automatically scanned for ocular, muscle, and jump artifacts using the Fieldtrip software (Oostenveld et al., 2011). Afterwards, artifacts were visually confirmed by a MEG expert. We segmented the remaining artifact-free data in 4-s segments (trials) and used then in an independent component analysis-based procedure to remove the heart artifact. At least 20 clean 4-s-long trials ( $80 \mathrm{~s}$ of brain activity) were then obtained from each participant, and 20 of these clean trials were randomly selected from each subject to equalize the number of trials. Previously to source data calculation, MEG time series were filtered into theta (4$8 \mathrm{~Hz})$, alpha $(8-12 \mathrm{~Hz})$, beta $(12-30 \mathrm{~Hz})$, and gamma $(30-45 \mathrm{~Hz})$ frequency bands with a 1,500 order finite impulse response filter designed using Hamming window and a two-pass filtering procedure.

\section{Source Reconstruction}

A regular volumetric grid with $10 \mathrm{~mm}$ spacing was created in MNI space. This set of nodes was transformed to each 
participant's space using a non-linear normalization between the native $\mathrm{T} 1$ image (whose coordinate system was previously converted to match the MEG coordinate system) and a standard T1 in MNI space. The forward model was solved using the realistic single-shell model introduced by Nolte (2003). Sources reconstruction was performed independently for each subject and frequency band, using a linearly constrained minimum variance (LCMV) beamformer (Van Veen et al., 1997). In order to combine the two types of sensors in the MEG scan, the leadfields were normalized by sensor type, and the same scaling factor was applied to the data. Beamformer filters were estimated from the average covariance of the trials using a $15 \%$ regularization factor. The neural MEG source-space time series were anatomically parcellated by dividing the cortex into 90 regions of interest (ROIs) according to the AAL atlas (TzourioMazoyer et al., 2002). After excluding the cerebellum, basal ganglia, thalamus, amygdala, insula and olfactory cortices, the anatomical model consisted of 72 ROIs. Per each one of them, we selected the first component, obtained with a principal component analysis (PCA), as the representative time series. Finally, the FC was assessed using the phase locking value (PLV), a phase synchronization measure that evaluates the distribution of phase differences extracted from each two ROIs time series (Mormann et al., 2000).

\section{Statistical Analysis}

The procedure for statistical comparisons relied on the network based statistics (NBS) introduced by Fornito et al. (2016) and Zalesky et al. (2010), which was carried out independently for each frequency band. The aim of this piece of work was the detection of any robust significant connected subnetworks (assembly of nodes for which a path can be found linking any pair of nodes in that subnetwork). These subnetworks consisted of several significant links ( $p$-value of 0.005 , ANCOVA test with age as covariate), which systematically should show either diminished or enhanced FC in the Val/Met group compared with the Val/Val homozygotes. Network-statistics was assessed through the sum of all F-values corresponding with the subnetwork's links. Candidate subnetworks were required to have a minimum size defined by the obligation of involving at least the $10 \%$ of the ROIs of the model (i.e., 7 ROIs for our atlas model). Then, to control for the multiple comparisons problem, the entire analysis pipeline was repeated 5,000 times after shuffling the original group's labels. At each repetition, the maximum statistic of the surrogate subnetworks was kept creating a maximal null distribution that ensured control of the family-wise error rate (FWER) at the subnetwork level. The network-statistics over each subnetwork in the original data set, was compared with the same measure in the randomized data. The NBS $p$-value represented the proportion of the permutation distribution with network-statistic values greater or equal than the network-statistic value of the original data. Only those subnetworks that survived after the NBS were used for the subsequent analyses as potential "MEG markers." As descriptive values for each significant subnetwork, we computed their average FC (across all links that belong to the subnetwork, i.e., their corresponding strength) values. These data were then used in the exploratory analysis for the correlation (Spearman's correlation) with the neuropsychological scores. In addition, for these strength values, we computed between-group statistical analysis using an ANCOVA with age as covariate. Results were not corrected for the multiple comparisons associated with the assessment of between-group differences in functional brain network for 5 frequency bands. Finally, to rank network's links based on their accuracy to distinguish between both groups, a logistic regression classification analysis with a leave-oneout cross-validation procedure was performed assessing the subnetwork-representative strength and the FC values of the individual links (see López et al., 2014). For classification purposes, Val/Met group was considered deleterious. Accuracy value represents the fraction of participants correctly classified. Sensitivity and specificity values represent the fractions of $\mathrm{Val} / \mathrm{Met}$ and Val/Val individuals correctly classified, respectively. Finally, positive predictive values (PPVs) and negative predictive values (NPVs) represent the fraction of individuals classified as $\mathrm{Val} / \mathrm{Met}$ that were really $\mathrm{Val} / \mathrm{Val}$ and the fraction of participants classified as Val/Val that were really Val/Met. Statistical analyses were carried out using Matlab R2016a (Mathworks Inc).

\section{RESULTS}

\section{Participant Data}

Table 1 shows the characteristics of the participants. There were no significant $(p>0.05)$ differences in age, educational attainment, neuropsychological performance or hippocampal/entorhinal volumes (Table 1).

Results of the FC analysis are summarized in Figures 1, 2, and Table 2. The network-based FC methodology pointed out the existence of an altered brain network composed by longrange connections between antero-posterior ROIs, showing a significant diminished FC in Val/Met participants when compared to Val/Val homozygotes. This alteration was restricted to the gamma frequency band.

Gamma subnetwork (CBPT $p=0.041$ ) comprised nine significant links (Figures 1, 2 and Table 2), with a clear anteroposterior pattern in which both (left and right) lateral superior occipital ROIs (l- and rLSOcc) connected with different frontal ROIs. The pattern of connections of the subnetwork (Figure 2) showed that both LSOcc ROIs played a role as hubs in two different sub-networks, uniquely connected to each other through the left gyrus rectus ROI. The ILSOcc connected with the right supplementary motor area (rMotor), the right superior frontal gyrus (rSFG), the left gyrus rectus (lRectus), the left orbital superior frontal gyrus (ISFo), and the left anterior cingulate cortex (lACC). On the other hand, the rLSOcc connected with the right orbital inferior frontal gyrus (rIFGo), the right orbital superior frontal gyrus (rSFo) and both gyrus rectus.

\section{Correlations Analysis Between MEG FC Data and Neuropsychological Assessment}

To further elucidate the relevance of the MEG FC markers to assess cognitive performance, a set of exploratory correlation analyses between the MEG FC marker values and cognitive scores 


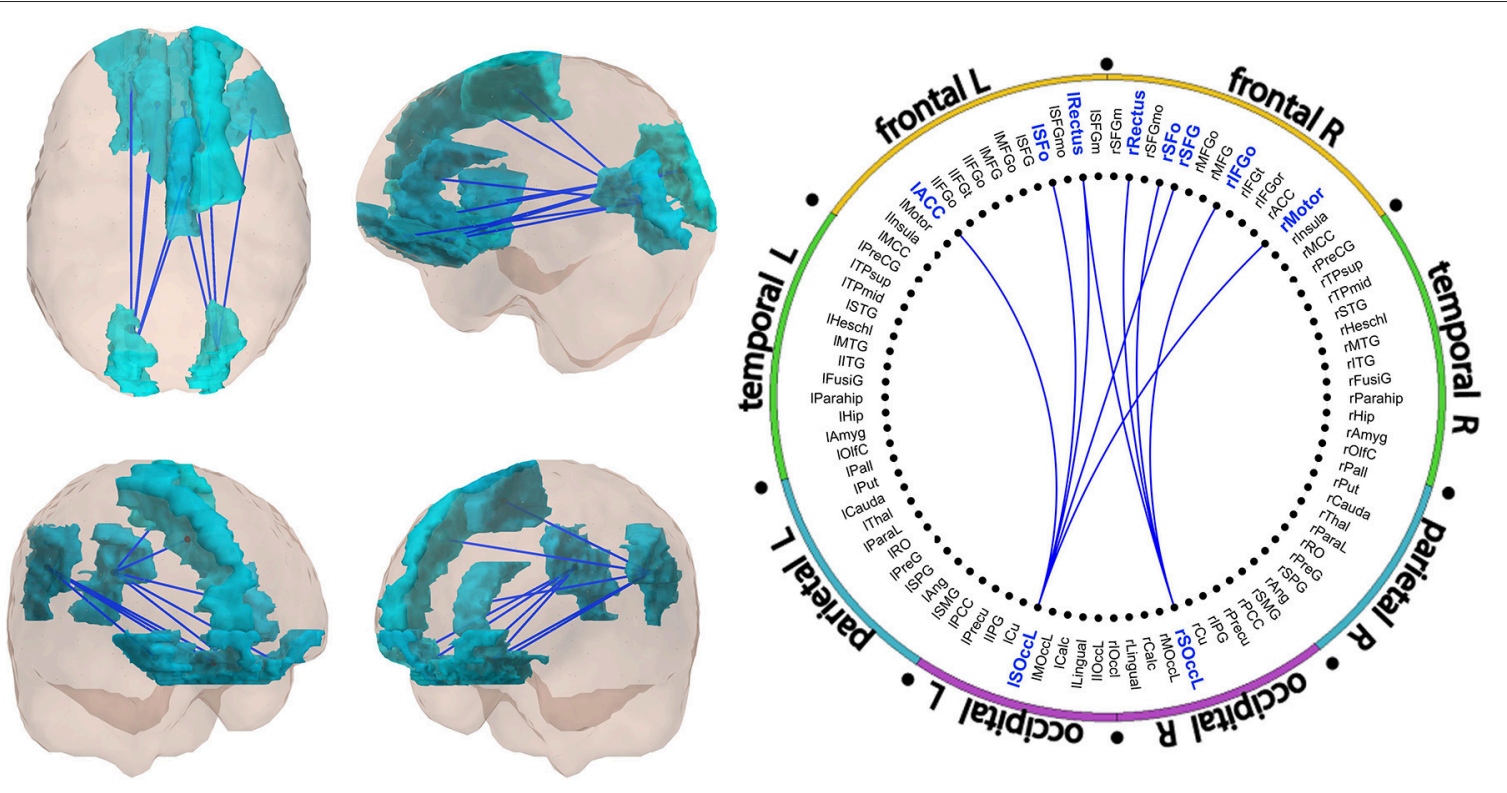

FIGURE 1 | Significant subnetwork in the gamma band. All links showed decreased FC in Val/Met individuals as compared to Val/Val participants. A schematic representation of the links is showed in Figure 2. FC values of the links are described in Table 2.

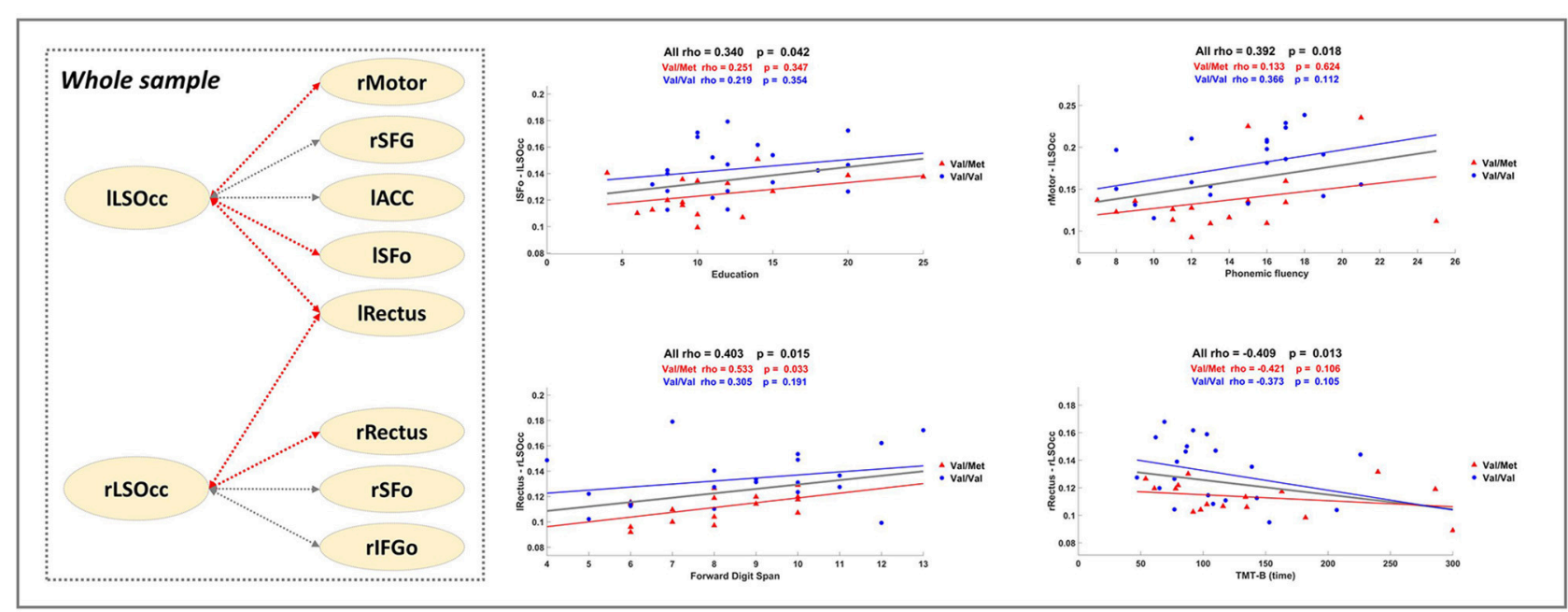

FIGURE 2 | Correlation Analysis. The dashed square in the left part presents a summary of the significant results described in Table 3. Gray dashed lines correspond to the links of the significant subnetwork. Red dashed lines indicate that the FC of the link is positively correlated with better performance. Scatter plots show some significant Spearman's rho values computed with all individuals within the sample (see bold highlighted values in Table 3). Blue and red lines depict, respectively, extra regression analysis carried out uniquely with Val/Nal and Val/Met groups (for information purposes).

were performed (Figure 2 and Table 3). Figure 2 displays a schematic representation of the significant subnetwork, where we have depicted the relationship between links and the results of the correlation analysis, which was carried out including the whole sample and showed a positive correlation between FC values and better cognitive performance (FDS, BDS PhF) and education. Age was negatively associated with the FC between rRectus and rLSOcc ROIs in the Val/Val group. A similar correlation was found in the Val/Met group for the FC between IRectus and
rLSOcc, although in this case the $p$-value was slightly above the threshold $(p<0.07)$.

\section{Classification}

In order to rank the FC results based on their capability to distinguish between both groups, a classification analysis was performed independently per every individual FC value found in the study, including links and subnetwork strength. The highest accuracy $(86.1 \%)$ was obtained for the subnetwork strength 
TABLE 2 | Functional connectivity results.

\begin{tabular}{|c|c|c|c|c|c|c|c|c|c|c|}
\hline & FC Val/Val & FC Val/Met & Statistics ( $p$-value) & \multicolumn{7}{|c|}{ Classification } \\
\hline ILSOcc-rSFG & $0.159 \pm 0.034$ & $0.129 \pm 0.020$ & 0.004026 & 0.722 & 0.750 & 0.700 & 0.667 & 0.778 & 0.858 & 0.548 \\
\hline ILSOcc-ISFo & $0.143 \pm 0.020$ & $0.124 \pm 0.015$ & 0.003335 & 0.611 & 0.625 & 0.600 & 0.556 & 0.667 & 0.769 & 0.435 \\
\hline ILSOcc-rMotor & $0.178 \pm 0.036$ & $0.137 \pm 0.040$ & 0.003255 & 0.694 & 0.813 & 0.600 & 0.619 & 0.800 & 0.837 & 0.519 \\
\hline ILSOcC-IACC & $0.186 \pm 0.041$ & $0.145 \pm 0.036$ & 0.003522 & 0.694 & 0.813 & 0.600 & 0.619 & 0.800 & 0.837 & 0.519 \\
\hline rLSOcc-rSFo & $0.129 \pm 0.021$ & $0.110 \pm 0.011$ & 0.001246 & 0.722 & 0.813 & 0.650 & 0.650 & 0.813 & 0.858 & 0.548 \\
\hline rLSOcc-rIFGo & $0.127 \pm 0.019$ & $0.111 \pm 0.007$ & 0.003521 & 0.750 & 0.875 & 0.650 & 0.667 & 0.867 & 0.879 & 0.578 \\
\hline rLSOcc-IRectus & $0.134 \pm 0.022$ & $0.111 \pm 0.011$ & 0.000651 & 0.806 & 0.875 & 0.750 & 0.737 & 0.882 & 0.918 & 0.640 \\
\hline rLSOcc-rRectus & $0.131 \pm 0.022$ & $0.113 \pm 0.012$ & 0.003927 & 0.667 & 0.750 & 0.600 & 0.600 & 0.750 & 0.814 & 0.490 \\
\hline
\end{tabular}

Functional Connectivity (FC) values are presented as mean \pm SD. Statistical analyses were performed using one-way ANCOVA (age as confounding covariate). The accuracy, sensitivity and specificity, negative predictive value (NPV) and positive predictive value (PPV) scores were obtained through a logistic regression analysis with leave-one-out cross-validation procedure. The confidence interval (Upper and Lower B) (Cl) for the statistic, was calculated using the $\beta$ approach (in bold, the highest scores of each variable).

I/r-LSOcc, left/right lateral superior occipital lobe; rMotor, right supplementary motor area; rSFG, right superior frontal gyrus; I/r-Rectus, left/right gyrus rectus; I/r-SFo, left/right orbital superior frontal gyrus; IACC, left anterior cingulate gyrus; rIFGo, right orbital inferior frontal gyrus.

TABLE 3 | FC markers correlation analysis.

\begin{tabular}{lccccc}
\hline & $\begin{array}{c}\text { ILSOcc- } \\
\text { ISFo }\end{array}$ & $\begin{array}{c}\text { ILSOcc- } \\
\text { rMotor }\end{array}$ & $\begin{array}{c}\text { ILSOcc- } \\
\text { IRectus }\end{array}$ & $\begin{array}{c}\text { rLSOcc- } \\
\text { IRectus }\end{array}$ & $\begin{array}{c}\text { rLSOcc- } \\
\text { rRectus }\end{array}$ \\
\hline Age & & & & $\left(-0.48^{*}\right)$ & {$[-0.51]$} \\
Education & $\mathbf{0 . 3 4}$ & & & & \\
Forward digit Span & & & & $\mathbf{0 . 4 0}$ & \\
& & & & $\mathbf{0 . 5 3 )}$ & \\
Backward digit & & & 0.33 & 0.33 & 0.34 \\
Span & & & & \\
Phonemic fluency & & $\mathbf{0 . 3 9}$ & {$[0.50]$} & & \\
TMT-B (time) & & & & & $-\mathbf{0 . 4 1}$ \\
\hline
\end{tabular}

Correlation analysis (Spearman rho values significant at $p<0.05$ ) are shown for each FC marker. Rho values are presented on basis of the sample used in the analysis: All sample, Nal/Met sample) or [ Val/Nal sample].

TMT-B, Trail-Making Test part B; I/r-SOccL, left/right superior occipital lobe; rMotor, right Supplementary motor area; $r S F G$, right superior frontal gyrus; I/r-Rectus, left/right gyrus rectus; I/r-SFo, left/right orbital superior frontal gyrus.

Significant correlations are depicted in Figure $\mathbf{2}$.

${ }^{*}$ Result with $0.05<p<0.06$.

Bold highlighted results are shown in Figure 2.

value, followed by the result of the lRectus-rLSOcc link (80.6\%), which supports our approach of focusing on networks rather than individual links. All the classification results are shown in Table 2.

\section{DISCUSSION}

In this research, we have carried out a MEG FC analysis to examine the influence of BDNF Val66Met SNP in CI older females. We found that Met allele carriers showed a diminished antero-posterior gamma band FC, which might be caused by a reduction in the ability to synchronize corresponding brain areas as accurately as Val/Val participants
(Beste et al., 2010). It has been reported that gamma oscillations mainly emerge from the coordinated interaction between excitatory-inhibitory neuronal activity (Buzsáki and Wang, 2012). In fact, GABAergic interneurons (i.e., parvalbuminpositive interneurons) are the main generators of gamma oscillations (Başar, 2013). In silico models of GABAergic network impairment (e.g., reduced GABAergic innervation or connectivity, or a long decay time of the inhibitory activity), together with studies conducted in AD patients, evidenced a dramatic reduction in the gamma band activity in this population (Stam et al., 2002; Başar, 2013; Başar et al., 2016; Metzner et al., 2016). On top of that, BDNF has also been found to influence GABAergic interneurons (Holm et al., 2009), and, as a synaptic trophic factor strengthening connections between brain networks, modulates gamma oscillations (Tamura et al., 2017). Therefore, the Met allele carriage apparently weakens this excitatory-inhibitory connection by reducing the amplitude and synchronization of gamma brain waves. Furthermore, the observed FC disruption involves several long-range connections (e.g., the lRectus-rLSOcc with an $80.6 \%$ of classification), demonstrating that synchronization in this frequency band has a role not only in local, but also in long-distance networks (Başar, 2013).

Similarly, it should be noted that the alteration of gamma oscillations in resting-state within the $\mathrm{AD}$ continuum is still controversial and little explored. Different studies have reported conflicting results showing increased or decreased gamma band activity (Stam et al., 2002; Stam, 2010; Wang et al., 2017). These discrepancies may be due, among other factors, to the severity of the disease and the experimental conditions applied (i.e., task vs. resting-state) (Wang et al., 2017). For example, gamma activity (both spontaneous or evoked/induced), has been located in many cortical brain areas (Fries, 2009) being involved in multiple cognitive functions (Başar, 2013; Başar et al., 2015). In addition, Buzsáki and Wang (Buzsáki and Wang, 2012) have stated that the activation of this oscillatory rhythm could vary 
depending on the underlying mechanism considered. On the other hand, the Met allele mutation in the $5^{\prime}$ pro-region of the BDNF protein (possibly leading to an excessive proBDNF production which could be toxic to neurons), produces an imbalance in the correct release of the mature BDNF peptide (Egan et al., 2003; Chen et al., 2004). Besides, BDNF expression is equitably ubiquitous throughout the brain (Zhang et al., 2007), and its receptors are abundantly expressed in parvalbuminpositive interneurons, which are selectively regulated by binding to the mature form of BDNF (Holm et al., 2009). As previously said, these GABAergic interneurons are crucial for the emergence of the gamma band oscillatory response (Zheng et al., 2011 as cited by Tamura et al., 2017). Thus, it may be that due to this functional mutation, the proBDNF is not only unable to adequately modulate GABAergic activity (Holm et al., 2009), but also to inhibit BDNF's availability, contributing to a potential neurobiological loss of function (Uegaki et al., 2017). All these evidences support the hypo-synchronized gamma band pattern found in the Met carrier group, highlighting that $B D N F$ Val66Met SNP may exert an influence on brain connectivity in healthy and pathological aging.

Regarding the relationship between significant FC results and neuropsychological scores, correlation analyses indicated that enhanced FC in the gamma subnetwork was associated with better cognitive performance (i.e., FDS, BDS and PhF) in the whole sample, but, as can be observed in the regression scatter plots (Figure 2), Val/Val homozygotes showed on average more FC. In line with this result, we would like to underscore the positive correlation between the ISFo-lLSOcc FC and education. Education is a well-established proxy of cognitive reserve (CR) (Stern, 2012), and a recent study in CI older adults across a 36month follow-up period concluded that CR-related differences in executive function decreased in both $\mathrm{Val} / \mathrm{Val}$ and $\mathrm{Val} / \mathrm{Met}$ participants, but became more notable in Met carriers (Ward et al., 2017). In our study, both groups showed a similar rho value when the correlation between ISFo-lLSOcc FC and education was performed for each group separately (Figure 2). However, the fact that the two groups behaved similarly and given the positive correlations between FC and cognitive performance, it could be suggested that the antero-posterior network activity differences found in our sample could be interpreted as a signature of the underlying mechanisms involved in CR. Following Ward et al. (2017) findings, we would expect that the strength of the correlation between FC and the cognitive status will deteriorate to a larger extent for Val/Met participants, constituting a downstream marker of aging-related neurodegeneration. Finally, the negative association between the rRectus-rLSOcc FC and age within the Val/Val group (not achieving statistical significance for the Val/Mets) supports the evidence that BDNF levels decrease throughout the aging process (Mattson and Magnus, 2006; Sohrabji and Lewis, 2006) and, therefore, another possible mechanism of greater susceptibility to age-related cognitive impairment.

From our point of view, these results suggest that electrophysiological resting-state FC, as measured by MEG, could be potentially considered as a non-invasive, sensitive biomarker enabling the assessment of brain functional integrity associated with genetic factors (Schofield et al., 2009; Voineskos et al., 2011; Papenberg et al., 2015; Chiesa et al., 2017), such as the BDNF Val66Met SNP. FC studies like the present one are expected to deepen the understanding of the pathophysiological processes underlying healthy and pathological aging (Babiloni et al., 2016; Walsh et al., 2017).

\section{LIMITATIONS AND FUTURE APPROACHES}

The evidence presented here emphasizes the relevance of the neuroimaging genetics field. However, there are several methodological issues that should be considered and that, as in many other cases could account for the conflicting results found through the literature on BDNF Val66Met SNP (for more information see Notaras and Hill, 2015). For example, the effect of the Met/Met genotype could not be appropriately examined (Zdanys et al., 2009; Forde et al., 2014). This is an important issue because the reduced dependent release of BDNF could vary from $18 \%$ for one Met allele to $29 \%$ in the Met/Met homozygous cases (Chen et al., 2006). In addition, analyzing additive or gene-gene interactions (Kauppi et al., 2014; Kennedy et al., 2014), ethnicity (Brooks et al., 2014; Lin et al., 2014), other BDNF's SNPs (e.g., rs56164415 or rs2072446) (Matyi et al., 2017), their epigenetic modulation, and gender differences, could also contribute to clarify BDNF's role in human brain functioning and neurodegeneration (Boulle et al., 2012; Honea et al., 2013).

Besides, we only selected CI older females who were APOE $\varepsilon 3 \varepsilon 3$, not including $A P O E \& 4$ allele carriers, neither its interaction with BDNF Val66Met SNP. Anyway, a study conducted by Sen and co-workers found that APOE $\varepsilon 3$ had little or no effect on BDNF levels (Sen et al., 2015 as cited by Fisher et al., 2018). This supports, on the other hand, the suitability of the selected study sample, since our results could be more purely restricted to the BDNF Val66Met SNP effects. In addition, while several studies have reached the conclusion that age-related pathological changes in brain structure and function could occur independently of genetic influence (Erickson et al., 2012), others claim that the genetic harmful effects may emerge as a factor of age (i.e., the antagonistic pleiotropy theory of aging) (Williams, 1957; Li et al., 2010; Voineskos et al., 2011). Therefore, the implementation of longitudinal studies could be an interesting way to shed light on how different genetic variations could affect different brain disorders throughout the entire life cycle (Gruber et al., 2012; Papenberg et al., 2015).

Finally, three more concerns should be highlighted. The first one is related to the non-existence of hippocampal volume differences between groups as reported in Lim et al. (2013, 2014a) and Rabl et al. (2014). We would like to clarify that Lim et al. (2013) were the only ones focused on a cognitively intact older adult population. However, that study was longitudinal, with a higher sample size than ours $(N=165)$, without contemplating sex differences and where $A \beta$ levels were also taken into account. Thus, we believe that none of the cited references challenge our results. Secondly, and regarding the analytical procedure, another possible limitation, particularly affecting low frequency 
bands results, would be the selection of $4 \mathrm{~s}$ length epochs. Longer epochs would increase the frequency-associated reliability, but we decided to keep $4 \mathrm{~s}$, based on previous studies of our group and the good trade-off between number of clean epochs and frequency resolution (Nakamura et al., 2017; Dimitriadis et al., 2018). Thirdly, we are aware that the FC metric (PLV) could be theoretically affected by source leakage. However, we believe that this is not the case for our results since: (a) the analysis conducted consisted on a between-groups comparison. This approach reduces the possibility of having false results driven by source leakage as this problem would be affecting both groups equally and (b) the subnetwork described in this paper was composed by long distance links, which are less likely to be affected by this issue.

\section{CONCLUSIONS}

This study demonstrates that multimodal analyses, combining MEG FC and genetics, offer valuable information in the assessment of possible selective brain network vulnerabilities associated with the beginning of the complex continuum from healthy to pathological aging. Furthermore, the noninvasiveness of MEG may be also useful as a surrogate endpoint for monitoring the effects of future pharmacological and non-pharmacological treatment interventions.

\section{REFERENCES}

Adamczuk, K., De Weer, A.-S., Nelissen, N., Chen, K., Sleegers, K., Bettens, K., et al. (2013). Polymorphism of brain derived neurotrophic factor influences $\beta$ amyloid load in cognitively intact apolipoprotein E $\varepsilon 4$ carriers. NeuroImage. Clin. 2, 512-520. doi: 10.1016/j.nicl.2013.04.001

Babiloni, C., Lizio, R., Marzano, N., Capotosto, P., Soricelli, A., Ivano, A., et al. (2016). Brain neural synchronization and functional coupling in Alzheimer' s disease as revealed by resting state EEG rhythms. Int. J. Psychophysiol. 103, 88-102. doi: 10.1016/j.ijpsycho.2015.02.008

Bai, F., Shu, N., Yuan, Y., Shi, Y., Yu, H., Wu, D., et al. (2012). Topologically convergent and divergent structural connectivity patterns between patients with remitted geriatric depression and amnestic mild cognitive impairment. J. Neurosci. 32, 4307-4318. doi: 10.1523/JNEUROSCI.5061-11.2012

Barnes, L. L., Wilson, R. S., Bienias, J. L., Schneider, J. A., Evans, D. A., and Bennett, D. A. (2005). Sex differences in the clinical manifestations of Alzheimer disease pathology. Arch. Gen. Psychiatry 62, 685-691. doi: 10.1001/archpsyc.62.6.685

Başar, E. (2013). A review of gamma oscillations in healthy subjects and in cognitive impairment. Int. J. Psychophysiol. 90, 99-117. doi: 10.1016/j.ijpsycho.2013.07.005

Başar, E., Durusu, D., Güntekin, B., and Yener, G. G. (2016). Delay of cognitive gamma responses in Alzheimer's disease. Neuroimage. Clin. 11, 106-115. doi: 10.1016/j.nicl.2016.01.015

Başar, E., and Düzgün, A. (2016). How is the brain working ? Research on brain oscillations and connectivities in a new "Take-Off” state. Int. J. Psychophysiol. 103, 3-11. doi: 10.1016/j.ijpsycho.2015.02.007

Başar, E., Tülay, E., and Güntekin, B. (2015). Multiple gamma oscillations in the brain: a new strategy to differentiate functional correlates and P300 dynamics. Int. J. Psychophysiol. 95, 406-420. doi: 10.1016/j.ijpsycho.2015.01.013

Bath, K. G., and Lee, F. S. (2006). Variant BDNF (Val66Met) impact on brain structure and function. Cogn. Affect. Behav. Neurosci. 6, 79-85. doi: 10.3758/CABN.6.1.79

Benton, A., and Hamsher, K. (1989). Multilingual aphasia examination, 2nd Edn. Iowa City, IA: Department of Neurology and Psychology.

Beste, C., Kolev, V., Yordanova, J., Domschke, K., Falkenstein, M., Baune, B. T., et al. (2010). The role of the BDNF Val66Met polymorphism

\section{AUTHOR CONTRIBUTIONS}

ICR-R, PC, and ML designed the final study. PM, MM-P, AM, and $\mathrm{RL}-\mathrm{H}$ were in charge of the initial selection of subjects and the neuropsychological part of the manuscript. $A B$ performed the genetic determinations. PC, ICR-R, ML, JdF-L, RB, EP, AF, and FM contributed to the MEG analyses and interpretation of data. ICR-R and PC drafted the manuscript and all authors revised and approved the final version.

\section{ACKNOWLEDGMENTS}

This study was supported by the project: PSI2009-14415C03-01 from the Spanish Ministry of Science and Economy and the project PI10/01585 from the National Institute of Health Carlos III. Complementary, it was supported by predoctoral fellowships from the Spanish Ministry of Education, Culture and Sports to ICR-R (FPU13/02064) and to RB (FPU13/06009), a predoctoral fellowship from La Caixa Foundation to JdF-L, and a postdoctoral fellowship from the Spanish Ministry of Economy and Competitiveness to PC (FJCI-2015-26755) and to a postdoctoral fellowship from the University Complutense of Madrid to ML. EP acknowledges the support of the MINECO through the grant TEC201680063-C3-2-R.

for the synchronization of error-specific neural networks. J. Neurosci. 30, 10727-10733. doi: 10.1523/JNEUROSCI.2493-10.2010

Binder, D. K., and Scharfman, H. E. (2004). Brain-derived neurotrophic factor. Growth Factors 22, 123-131. doi: 10.1080/08977190410001723308

Boulle, F., van den Hove, D. L. A., Jakob, S. B., Rutten, B. P., Hamon, M., van Os, J., et al. (2012). Epigenetic regulation of the BDNF gene: implications for psychiatric disorders. Mol. Psychiatry 17, 584-596. doi: 10.1038/mp.2011.107

Brooks, S. J., Nilsson, E. K., Jacobsson, J. A., Stein, D. J., Fredriksson, R., Lind, L., et al. (2014). BDNF polymorphisms are linked to poorer working memory performance, reduced cerebellar and hippocampal volumes and differences in prefrontal cortex in a Swedish elderly population. PLoS ONE 9:e82707. doi: 10.1371/journal.pone.0082707

Buzsáki, G., and Wang, X.-J. (2012). Mechanisms of gamma oscillations. Annu. Rev. Neurosci. 35, 203-225. doi: 10.1146/annurev-neuro-062111-150444

Chen, A., Xiong, L.-J., Tong, Y., and Mao, M. (2013). The neuroprotective roles of BDNF in hypoxic ischemic brain injury. Biomed. Rep. 1, 167-176. doi: 10.3892/br.2012.48

Chen, Z.-Y., Jing, D., Bath, K. G., Ieraci, A., Khan, T., Siao, C.-J., et al. (2006). Genetic Variant BDNF (Val66Met) Polymorphism alters anxiety-related behavior. Science 314, 140-143. doi: 10.1126/science.1129663

Chen, Z.-Y., Patel, P. D., Sant, G., Meng, C.-X., Teng, K. K., Hempstead, B. L., et al. (2004). Variant Brain-Derived Neurotrophic Factor (BDNF) (Met66) alters the intracellular trafficking and activity-dependent secretion of wild-type BDNF in neurosecretory cells and cortical neurons. J. Neurosci. 24, 4401-4411. doi: 10.1523/JNEUROSCI.0348-04.2004

Chiesa, P. A., Cavedo, E., Lista, S., Thompson, P. M., Hampel, H., and If, T. D. (2017). Revolution of resting-state functional neuroimaging genetics in Alzheimer' s disease. Trends Neurosci. 40, 469-480. doi: 10.1016/j.tins.2017.06.002

Cohen-Cory, S., and Fraser, S. E. (1995). Effects of brain-derived neurotrophic factor on optic axon branching and remodelling in vivo. Nature 378, 192-196. doi: $10.1038 / 378192 \mathrm{a} 0$

Criscuolo, C., Fabiani, C., Bonadonna, C., Origlia, N., and Domenici, L. (2015). BDNF prevents amyloid-dependent impairment of LTP in the entorhinal cortex by attenuating p38 MAPK phosphorylation. Neurobiol. Aging 36, 1303-1309. doi: 10.1016/j.neurobiolaging.2014.11.016 
Cuesta, P., Garcés, P., Castellanos, N. P., López, M. E., Aurtenetxe, S., Bajo, R., et al. (2015). Influence of the APOE $\varepsilon 4$ allele and mild cognitive impairment diagnosis in the disruption of the MEG resting state functional connectivity in sources space. J. Alzheimers Dis. 44, 493-505. doi: 10.3233/JAD-141872

Dimitriadis, S. I., López, M. E., Bruña, R., Cuesta, P., Marcos, A., Maest,ú, F., et al. (2018). How to build a functional connectomic biomarker for mild cognitive impairment from source reconstructed MEG Resting-state activity: The combination of ROI representation and connectivity estimator matters. Front. Neurosci. 12:306. doi: 10.3389/fnins.2018.00306

Dincheva, I., Glatt, C. E., and Lee, F. S. (2012). Impact of the BDNF Val66Met polymorphism on cognition. Neuroscientist 18, 439-451. doi: $10.1177 / 1073858411431646$

Egan, M. F., Kojima, M., Callicott, J. H., Goldberg, T. E., Kolachana, B. S., Bertolino, A., et al. (2003). The BDNF val66met polymorphism affects activitydependent secretion of BDNF and human memory and hippocampal function. Cell 112, 257-269. doi: 10.1016/S0092-8674(03)00035-7

Erickson, K. I., Miller, D. L., and Roecklein, K. A. (2012). The aging hippocampus: interactions between exercise, depression, and BDNF. Neuroscientist 18, 82-97. doi: $10.1177 / 1073858410397054$

Fischl, B., Salat, D. H., Busa, E., Albert, M., Dieterich, M., Haselgrove, C., et al. (2002). Whole brain segmentation. Neuron 33, 341-355. doi: 10.1016/S0896-6273(02)00569-X

Fisher, D. W., Bennett, D. A., and Dong, H. (2018). Sexual dimorphism in predisposition to Alzheimer's disease. Neurobiol. Aging 70, 308-324. doi: 10.1016/j.neurobiolaging.2018.04.004

Fleisher, A., Grundman, M., Jack, C. R., Petersen, R. C., Taylor, C., Kim, H. T., et al. (2005). Sex, apolipoprotein E $\varepsilon 4$ status, and hippocampal volume in mild cognitive impairment. Arch. Neurol. 62:953. doi: 10.1001/archneur.62.6.953

Folstein, M. F., Folstein, S. E., and McHugh, P. R. (1975). \&quot;Mini-mental state\&quot;. A practical method for grading the cognitive state of patients for the clinician. J. Psychiatr. Res. 12, 189-198. doi: 10.1016/0022-3956(75)90026-6

Forde, N. J., Ronan, L., Suckling, J., Scanlon, C., Neary, S., Holleran, L., et al. (2014). Structural neuroimaging correlates of allelic variation of the BDNF val66met polymorphism. Neuroimage 90, 280-289. doi: 10.1016/j.neuroimage.2013.12.050

Fornito, A., Zalesky, A., and Bullmore, E. T. (2016). Fundamentals of Brain Network Analysis. London: Elsevier.

Fries, P. (2009). Neuronal gamma-band synchronization as a fundamental process in cortical computation. Annu. Rev. Neurosci. 32, 209-224. doi: 10.1146/annurev.neuro.051508.135603

Gruber, O., Hasan, A., Scherk, H., Wobrock, T., Schneider-Axmann, T., Ekawardhani, S., et al. (2012). Association of the brain-derived neurotrophic factor val66met polymorphism with magnetic resonance spectroscopic markers in the human hippocampus: in vivo evidence for effects on the glutamate system. Eur. Arch. Psychiatry Clin. Neurosci. 262, 23-31. doi: 10.1007/s00406-011-0214-6

Hashimoto, R., Hirata, Y., Asada, T., Yamashita, F., Nemoto, K., Mori, T., et al. (2009). Effect of the brain-derived neurotrophic factor and the apolipoprotein E polymorphisms on disease progression in preclinical Alzheimer's disease. Genes. Brain. Behav. 8, 43-52. doi: 10.1111/j.1601-183X.2008.00440.x

Holm, M. M., Nieto-gonzalez, J. L., Vardya, I., Vaegter, C. B., and Nykjaer, A. (2009). Mature BDNF, but not proBDNF, reduces excitability of fastspiking interneurons in mouse dentate gyrus. J. Neurosci. 29, 12412-12418. doi: 10.1523/JNEUROSCI.2978-09.2009

Honea, R. A., Cruchaga, C., Perea, R. D., Saykin, A. J., Burns, J. M., Weinberger, D. R., et al. (2013). Characterizing the role of brain derived neurotrophic factor genetic variation in Alzheimer's disease neurodegeneration. PLoS ONE8 8:e76001. doi: 10.1371/journal.pone.0076001

Horch, H. W., Krüttgen, A., Portbury, S. D., and Katz, L. C. (1999). Destabilization of cortical dendrites and spines by BDNF. Neuron 23, 353-364. doi: 10.1016/S0896-6273(00)80785-0

Huang, C. C., Liu, M. E., Chou, K. H., Yang, A. C., Hung, C. C., Hong, C. J., et al. (2014). Effect of BDNF Val66Met polymorphism on regional white matter hyperintensities and cognitive function in elderly males without dementia. Psychoneuroendocrinology 39, 94-103. doi: 10.1016/j.psyneuen.2013.09.027

Jang, J. H., Yun, J.-Y., Jung, W. H., Shim, G., Byun, M. S., Hwang, J. Y., et al. (2012). The impact of genetic variation in comt and bdnf on resting-state functional connectivity. Int. J. Imaging Syst. Technol. 22, 97-102. doi: 10.1002/ima.22000
Kambeitz, J. P., Bhattacharyya, S., Kambeitz-Ilankovic, L. M., Valli, I., Collier, D. A., and McGuire, P. (2012). Effect of BDNF val66met polymorphism on declarative memory and its neural substrate: a meta-analysis. Neurosci. Biobehav. Rev. 36, 2165-2177. doi: 10.1016/j.neubiorev.2012.07.002

Kaplan, E., Goodglass, H., and Weintraub, S. (1983). The Boston Naming Test. Philadelphia: Lea and Febiger.

Kauppi, K., Nilsson, L.-G., Persson, J., and Nyberg, L. (2014). Additive genetic effect of APOE and BDNF on hippocampus activity. Neuroimage 89, 306-313. doi: 10.1016/j.neuroimage.2013.11.049

Kennedy, K. M., and Raz, N. (2009). Aging white matter and cognition: differential effects of regional variations in diffusion properties on memory, executive functions, and speed. Neuropsychologia 47, 916-927. doi: 10.1016/j.neuropsychologia.2009.01.001

Kennedy, K. M., Reese, E. D., Horn, M. M., Sizemore, A. N., Unni, A. K., Meerbrey, M. E., et al. (2014). BDNF val66met polymorphism affects aging of multiple types of memory. Brain Res. 1612, 104-117. doi: 10.1016/j.brainres.2014.09.044

Li, S.-C., Chicherio, C., Nyberg, L., von Oertzen, T., Nagel, I. E., Papenberg, G., et al. (2010). Ebbinghaus revisited: influences of the BDNF Val 66 met polymorphism on backward serial recall are modulated by human aging. J. Cogn. Neurosci. 22, 2164-2173. doi: 10.1162/jocn.2009.21374

Lim, Y. Y., Villemagne, V. L., Laws, S. M., Ames, D., Pietrzak, R. H., Ellis, K. A., et al. (2013). BDNF Val66Met, A $\beta$ amyloid, and cognitive decline in preclinical Alzheimer's disease. Neurobiol. Aging 34, 2457-2464. doi: 10.1016/j.neurobiolaging.2013.05.006

Lim, Y. Y., Villemagne, V. L., Laws, S. M., Ames, D., Pietrzak, R. H., and Ellis, K. A., et al. (2014a). Effect of BDNF Val66Met on memory decline and hippocampal atrophy in prodromal alzheimer's disease: a preliminary study. PLoS ONE 9:e86498. doi: 10.1371/journal.pone.0086498.eCollection2014

Lim, Y. Y., Villemagne, V. L., Laws, S. M., Pietrzak, R. H., Snyder, P. J., Ames, D., et al. (2014b). APOE and BDNF polymorphisms moderate amyloid $\beta$ related cognitive decline in preclinical Alzheimer's disease. Mol. Psychiatry 20, 1322-1328. doi: 10.1038/mp.2014.123

Lin, Y., Cheng, S., Xie, Z., and Zhang, D. (2014). Association of rs6265 and rs2030324 polymorphisms in brain-derived neurotrophic factor gene with Alzheimer's disease: a meta-analysis. PLoS ONE 9:e94961. doi: 10.1371/journal.pone.0094961

Lobo, A., Ezquerra, J., Gómez Burgada, F., Sala, J. M., and Seva Díaz, A. (1979). [Cognocitive mini-test (a simple practical test to detect intellectual changes in medical patients)]. Actas Luso. Esp. Neurol. Psiquiatr. Cienc. Afines 7, 189-202.

López, M. E., Bruña, R., Aurtenetxe, S., Pineda-Pardo, J. A., Marcos, A., Arrazola, J., et al. (2014). Alpha-band hypersynchronization in progressive mild cognitive impairment: a magnetoencephalography study. J. Neurosci. 34, 14551-14559. doi: 10.1523/JNEUROSCI.0964-14.2014

Marosi, K., and Mattson, M. P. (2014). BDNF mediates adaptive brain and body responses to energetic challenges. Trends Endocrinol. Metab. 25, 89-98. doi: 10.1016/j.tem.2013.10.006

Mattson, M. P., and Magnus, T. (2006). Ageing and neuronal vulnerability. Nat. Rev. Neurosci. 7, 278-294. doi: 10.1038/nrn1886

Mattson, M. P., Maudsley, S., and Martin, B. (2004). BDNF and 5-HT: a dynamic duo in age-related neuronal plasticity and neurodegenerative disorders. Trends Neurosci. 27, 589-594. doi: 10.1016/j.tins.2004.08.001

Matyi, J., Tschanz, J. T., Rattinger, G. B., Sanders, C., Vernon, E. K., Corcoran, C., et al. (2017). Sex differences in risk for Alzheimer's disease related to neurotrophin gene polymorphisms: the cache county memory study. J. Gerontol. Ser. A 72, 1607-1613. doi: 10.1093/gerona/glx092

Metzner, C., Schweikard, A., and Zurowski, B. (2016). Multifactorial modeling of impairment of evoked gamma range oscillations in schizophrenia. Front. Comput. Neurosci. 10:89. doi: 10.3389/fncom.2016.00089

Mormann, F., Lehnertz, K., David, P., and and, E., Elger, C. (2000). Mean phase coherence as a measure for phase synchronization and its application to the EEG of epilepsy patients. Phys. D Nonlinear Phenom. 144, 358-369. doi: 10.1016/S0167-2789(00)00087-7

Nakamura, A., Cuesta, P., Kato, T., Arahata, Y., Iwata, K., Yamagishi, M., et al. (2017). Early functional network alterations in asymptomatic elders at risk for Alzheimer's disease. Sci. Rep. 7:6517. doi: 10.1038/s41598-017-06876-8.

Neu, S. C., Pa, J., Kukull, W., Beekly, D., Kuzma, A., Gangadharan, P., et al. (2017). Apolipoprotein E genotype and sex risk factors for Alzheimer disease: a metaanalysis. JAMA Neurol. 74, 1178-1189. doi: 10.1001/jamaneurol.2017.2188 
Nolte, G. (2003). The magnetic lead field theorem in the quiasi-static approximation and its use for magnetoencephalography forward calculation in realistic volume conductors. Phys. Med. Biol. 48, 3637-3652. doi: $10.1088 / 0031-9155 / 48 / 22 / 002$

Notaras, M., and Hill, R. A. (2015). The BDNF gene Val66Met polymorphism as a modifier of psychiatric disorder susceptibility : progress and controversy The BDNF gene Val66Met polymorphism as a modi fi er of psychiatric disorder susceptibility : progress and controversy. Mol. Psychiatry 20, 916-30. doi: $10.1038 / \mathrm{mp} .2015 .27$.

Oostenveld, R., Fries, P., Maris, E., and Schoffelen, J.-M. (2011). FieldTrip: open source software for advanced analysis of MEG, EEG, and invasive electrophysiological data. Comput. Intell. Neurosci. 2011:156869. doi: $10.1155 / 2011 / 156869$

Papenberg, G., Salami, A., Persson, J., Lindenberger, U., and Bäckman, L. (2015). Genetics and functional imaging: effects of APOE, BDNF, COMT, and KIBRA in aging. Neuropsychol. Rev. 25, 47-62. doi: 10.1007/s11065-015-9279-8

Pievani, M., de Haan, W., Wu, T., Seeley, W. W., and Frisoni, G. B. (2011). Functional network disruption in the degenerative dementias. Lancet Neurol. 10, 829-843. doi: 10.1016/S1474-4422(11)70158-2

Pievani, M., Filippini, N., van den Heuvel, M. P., Cappa, S. F., and Frisoni, G. B. (2014). Brain connectivity in neurodegenerative diseases-from phenotype to proteinopathy. Nat. Publ. Gr. 10, 1-14. doi: 10.1038/nrneurol.2014.178

Qin, X.-Y., Cao, C., Cawley, N. X., Liu, T.-T., Yuan, J., Loh, Y. P., et al. (2017). Decreased peripheral brain-derived neurotrophic factor levels in Alzheimer's disease: a meta-analysis study $(\mathrm{N}=7277)$. Mol. Psychiatry 22, 312-320. doi: 10.1038/mp.2016.62

Rabl, U., Meyer, B. M., Diers, K., Bartova, L., Berger, A., Mandorfer, D., et al. (2014). Additive gene-environment effects on hippocampal structure in healthy humans. J. Neurosci. 34, 9917-9926. doi: 10.1523/JNEUROSCI.3113-13.2014

Reitan, R. M. (1958). Validity of the trail making test as an indicator of organic brain damage. Percept. Mot. Skills 8, 271-276. doi: 10.2466/pms.1958.8. 3.271

Rothman, S. M., and Mattson, M. P. (2013). Activity-dependent, stressresponsive BDNF signaling and the quest for optimal brain health and resilience throughout the lifespan. Neuroscience 239, 228-240. doi: 10.1016/j.neuroscience.2012.10.014

Schofield, P. R., Williams, L. M., Paul, R. H., Gatt, J. M., Brown, K., Luty, A., et al. (2009). Disturbances in selective information processing associated with the BDNF Val66Met polymorphism: evidence from cognition, the P300 and fronto-hippocampal systems. Biol. Psychol. 80, 176-188. doi: 10.1016/j.biopsycho.2008.09.001

Sen, A., Nelson, T. J., and Alkon, D. L. (2015). ApoE4 and A oligomers reduce BDNF expression via HDAC nuclear translocation. J. Neurosci. 35, 7538-7551. doi: 10.1523/JNEUROSCI.0260-15.2015

Sohrabji, F., and Lewis, D. K. (2006). Estrogen-BDNF interactions: implications for neurodegenerative diseases. Front. Neuroendocrinol. 27, 404-414. doi: 10.1016/j.yfrne.2006.09.003

Stam, C. J. (2010). Use of magnetoencephalography (MEG) to study functional brain networks in neurodegenerative disorders. J. Neurol. Sci. 289, 128-134. doi: 10.1016/j.jns.2009.08.028

Stam, C. J. (2014). Modern network science of neurological disorders. Nat. Rev. Neurosci. 15, 683-695. doi: 10.1038/nrn3801

Stam, C. J., van Cappellen van Walsum, A. M., Pijnenburg, Y. A. L., Berendse, H. W., de Munck, J. C., Scheltens, P., et al. (2002). Generalized synchronization of MEG recordings in Alzheimer's disease: evidence for involvement of the gamma band. J. Clin. Neurophysiol. 19, 562-574. doi: 10.1097/00004691-200212000-00010

Stern, Y. (2012). Cognitive reserve in ageing and Alzheimer's disease. Lancet Neurol. 11, 1006-1012. doi: 10.1016/S1474-4422(12)70191-6

Tamura, H., Shiosaka, S., and Morikawa, S. (2017). Neurochemistry International Trophic modulation of gamma oscillations : The key role of processing protease for Neuregulin-1 and BDNF precursors. Neurochem. Int. 119, 2-10. doi: 10.1016/j.neuint.2017.12.002

Taulu, S., and Simola, J. (2006). Spatiotemporal signal space separation method for rejecting nearby interference in MEG measurements. Phys. Med. Biol. 51, 1759-1768. doi: 10.1088/0031-9155/51/7/008

Tzourio-Mazoyer, N., Landeau, B., Papathanassiou, D., Crivello, F., Etard, O., Delcroix, N., et al. (2002). Automated anatomical labeling of activations in SPM using a macroscopic anatomical parcellation of the MNI MRI single-subject brain. Neuroimage 15, 273-289. doi: 10.1006/nimg.2001.0978
Uegaki, K., Kumanogoh, H., Mizui, T., and Hirokawa, T. (2017). BDNF binds its Pro-Peptide with high affinity and the common Val66Met polymorphism Attenuates the Interaction. Int. J. Mol. Sci. 18:1042. doi: 10.3390/ijms 18051042 Van Veen, B. D., van Drongelen, W., Yuchtman, M., and Suzuki, A. (1997). Localization of brain electrical activity via linearly constrained minimum variance spatial filtering. IEEE Trans. Biomed. Eng. 44, 867-880. doi: $10.1109 / 10.623056$

Varela, F., Lachaux, J., Rodriguez, E., and Martinerie, J. (2001). The brainweb: phase synchronization and large-scale integration. Nat. Rev. Neurosci. 2, 229-239. doi: 10.1038/35067550

Voineskos, A. N., Lerch, J. P., Felsky, D., Shaikh, S., Rajji, T. K., Miranda, D., et al. (2011). The brain-derived neurotrophic factor Val66Met polymorphism and prediction of neural risk for Alzheimer disease. Arch. Gen. Psychiatry 68, 198-206. doi: 10.1001/archgenpsychiatry.2010.194

Walsh, C., Drinkenburg, W. H. I. M., and Ahnaou, A. (2017). Neuroscience and biobehavioral reviews neurophysiological assessment of neural network plasticity and connectivity : progress towards early functional biomarkers for disease interception therapies in Alzheimer's disease. Neurosci. Biobehav. Rev. 73, 340-358. doi: 10.1016/j.neubiorev.2016.12.020

Wang, J., Fang, Y., Wang, X., Yang, H., and Yu, X. (2017). Enhanced gamma activity and cross-frequency interaction of electroencephalographic oscillations in patients with Alzheimer's disease. Front. Aging. Neurosci. 9:243. doi: 10.3389/fnagi.2017.00243

Ward, D. D., Andel, R., Saunders, N. L., Thow, M. E., Klekociuk, S. Z. Bindoff, A. D., et al. (2017). The BDNF Val66Met polymorphism moderates the effect of cognitive reserve on 36-month cognitive change in healthy older adults. Alzheimers Dement. Transl. Res. Clin. Interv. 3, 323-331. doi: 10.1016/j.trci.2017.04.006

Wechsler, D. (1997). Wechsler Memory Scale- Third Edition Manual. San Antonio, TX: The Psychological Corporation.

Wei, S.-M., Eisenberg, D. P., Kohn, P. D., Kippenhan, J. S., Kolachana, B. S., Weinberger, D. R., et al. (2012). Brain-derived neurotrophic factor Val66Met polymorphism affects resting regional cerebral blood flow and functional connectivity differentially in women versus men. J. Neurosci. 32, 7074-7081. doi: 10.1523/JNEUROSCI.5375-11.2012

Williams, G. C. (1957). Pleiotropy, natural selection, and the evolution of senescence. Evolution 11, 398-411.

Zalesky, A., Fornito, A., and Bullmore, E. T. (2010). Network-based statistic: identifying differences in brain networks. Neuroimage 53, 1197-1207. doi: 10.1016/j.neuroimage.2010.06.041

Zdanys, K. F., Kleiman, T. G., Zhang, H., Ozbay, F., MacAvoy, M. G., Gelernter, J., et al. (2009). BDNF variants, premorbid educational attainment, and disease characteristics in alzheimer's disease: an exploratory study. J. Alzheimers Dis. 17, 887-898. doi: 10.3233/JAD-2009-1106

Zhang, H.-T., Li, L.-Y., Zou, X.-L., Song, X.-B., Hu, Y.-L., Feng, Z.-T., et al. (2007). Immunohistochemical distribution of NGF, BDNF, NT-3, and NT4 in adult Rhesus monkey brains. J. Histochem. Cytochem. 55, 1-19. doi: 10.1369/jhc.6A6952.2006

Zheng, K., An, J. J., Yang, F., Xu, W., Xu, Z.-Q. D., Wu, J., et al. (2011). TrkB signaling in parvalbumin-positive interneurons is critical for gamma-band network synchronization in hippocampus. Proc. Natl. Acad. Sci. U.S.A. 108, 17201-17206. doi: 10.1073/pnas.1114241108

Ziegler, E., Foret, A., Mascetti, L., Muto, V., Le Bourdiec-shaffii, A., Stender, J., et al. (2013). Altered white matter architecture in BDNF Met carriers. PLoS ONE 8:e69290. doi: 10.1371/journal.pone.0069290

Conflict of Interest Statement: The authors declare that the research was conducted in the absence of any commercial or financial relationships that could be construed as a potential conflict of interest.

The handling Editor declared a shared affiliation, though no other collaboration, with one of the authors JdF-L.

Copyright (c) 2018 Rodríguez-Rojo, Cuesta, López, de Frutos-Lucas, Bruña, Pereda, Barabash, Montejo, Montenegro-Peña, Marcos, López-Higes, Fernández and Maestú. This is an open-access article distributed under the terms of the Creative Commons Attribution License (CC BY). The use, distribution or reproduction in other forums is permitted, provided the original author(s) and the copyright owner(s) are credited and that the original publication in this journal is cited, in accordance with accepted academic practice. No use, distribution or reproduction is permitted which does not comply with these terms. 